\title{
«Biblia y moral». Los criterios de interpretación en el documento de la Pontificia Comisión Bíblica (2008)
}

Gustavo Irrazábal*

\section{Resumen}

El presente trabajo es un estudio sobre los criterios de interpretación de los textos bíblicos propuestos por el documento de la Pontificia Comisión Bíblica, "Biblia y moral. Raíces bíblicas del comportamiento cristiano" (2008). El documento parte del concepto clave de "moral revelada", entendiendo por tal la iniciativa salvífica de Dios que suscita la respuesta religiosa y ética del ser humano. Queda preservada de este modo la originalidad de la moral bíblica, la cual, sin menoscabo de su continuidad con la moral natural, integra esta última en el contexto dialogal de la Alianza. A partir de este cuadro de referencia, el documento formula criterios de discernimiento moral capaces de orientar al intérprete en la tarea de elaboración, a la luz de los textos bíblicos, de normas concretas de conducta. Ello permite superar la tendencia a buscar en los textos bíblicos indicaciones normativas prescindiendo del contexto literario y de sentido en el que están insertas.

\footnotetext{
Abogado (UCA 1984). Sacerdote por la Arquidiócesis de Buenos Aires (7-12-1991). Bachiller en Teología (1991). Estudios de Licenciatura y Doctorado en Teología Moral, en la Pontificia Universidad Gregoriana de Roma (1999). Formador en el Seminario de Buenos Aires (2000-2006). Desde 2000, profesor de Teología Moral en la Facultad de Teología de la UCA, en los cursos de Moral Fundamental, Doctrina Social, Moral Especial (en particular, moral sexual y moral social y política) y Licenciatura en Teología Moral. Profesor de Doctrina Moral de la Iglesia en el Especialización en Derecho Penal de la Facultad de Derecho de la UCA, y de la Especialización en Doctrina Social de la Iglesia de la misma Universidad. Contacto: girrazabal@gmail.com
} 


\title{
Palabras clave
}

Biblia, discernimiento, normas, moral revelada, moral natural.

\section{«Bible and moral». Interpretation criteria in the document of the Pontifical Biblical Commission (2008)}

\begin{abstract}
This text is a study of the of interpretation criteria for biblical texts proposed by the document of the Pontifical Biblical Comission, "The Bible and Morality. Biblical Roots of Christian Behavior" (May 11 , 2008). This document starts from the clue-concept of "revealed morality", that is, God's saving initiative, which provokes the religious and ethical response of the human being. In this way, the originality of biblical morality is preserved, as well as the continuity with natural morality, which is integrated in the dialogical context of the Alliance. Within this reference framework, the document formulates criteria of discernment, in order to elaborate, in the light of biblical texts, concrete norms of behaviour. It also, makes it possible to overcome the tendency to look for ethical indications in the biblical texts without paying due attention to the literary context in which they are embedded.
\end{abstract}

\section{Keywords}

Bible, discernment, norms, moral disclosed, natural morality.

En este trabajo me propongo reflexionar sobre los criterios de interpretación de los textos bíblicos propuestos por el documento "Biblia y moral. Raíces bíblicas del comportamiento cristiano" (en 
adelante, ByM), elaborado por la Pontificia Comisión Bíblica (11 de mayo, 2008) ${ }^{1}$. Para ello consideraré, en primer lugar, el marco de referencia general del documento: su finalidad, sus presupuestos, sus objetivos y sus ideas-clave. A continuación, me referiré a los criterios en particular, con un especial énfasis en aquél que reviste a mi juicio una especial significación para la reflexión moral: el criterio del discernimiento. Finalmente, propondré dos ejemplos que ilustran las posibilidades que abren estos criterios en el recurso a los textos bíblicos, y esbozaré algunas conclusiones sobre el uso de la Sagrada Escritura en el campo moral, tanto por parte de la teología como del magisterio.

A lo largo de este recorrido, trataré de poner en evidencia el modo con que este documento recoge, sistematiza y a la vez profundiza una aproximación a la Sagrada Escritura que se ha ido perfilando en el ámbito de la teología moral, tras los ásperos debates posteriores al Concilio Vaticano II. Por otro lado, no sería posible dejar de señalar ciertas ambigüedades del texto, debidas probablemente al hecho de que, tratándose de un tema que exige una estrecha colaboración entre exegetas y teólogos, estos últimos parecen haber estado ausentes en su elaboración ${ }^{2}$. Ello podría haber dificultado una comprensión más

La versión oficial del documento ha sido redactada solo en francés e italiano. Por mi parte, cito la traducción al español de Luis H. Rivas (Buenos Aires: Ágape, 2011). Siglas: ByM: Biblia y Moral; DV: Dei Verbum; VS: Veritatis Splendor.

"En sí misma ésta (ética bíblica) es una tarea compleja para la cual son pocos los que están bien preparados; aquellos que son especialistas en ética generalmente carecen de un entrenamiento intensivo y adecuado en estudios bíblicos, y aquellos que son especialistas en estudios bíblicos con frecuencia carecen de la sofisticación en la reflexión ética", James Gustafson, "The Changing Use of the Bible in Christian Ethics", en Charles Curran y Richard McCormick (coord.), Readings in Moral Theology, vol. 4: The Use of Scripture in Moral Theology (New York: Paulist Press, 1984), 151. La falta de un trabajo interdisciplinar puede inferirse a partir de comentarios de miembros de la Pontificia Comisión Bíblica. Cf. Olivier Artus, "Bible et morale. Quels critères pour discerner? Le document de 2008 de la Commission biblique pontificale", Revue d'étique et de théologie morale 260 (2009): 51. Lo mismo surge de la presentación del documento hecha por el Secretario de este organismo. Cf. Klemens Stock, "Biblia y Moral. Las raíces bíblicas del actuar cristiano. El último documento de la Pontificia Comisión Bíblica", Lumen 59 (2010): 16. De todos modos, esta idea de colaboración en dos etapas (subentrando el moralista en la segunda de ellas) no es nueva. Cf. Pablo VI, Discurso a los profesores italianos de Sagrada Escritura, XXII Semana Bíblica Nacional, septiembre 29, 1972. www.vatican.va/holy_father/paul_vi/speeches/1972/september/documents/hf_p-vi_spe_19720929_ xxii-settimana-biblica_it.html. 
exacta de los problemas que subyacen en el recurso a cierto vocabulario, y de los alcances de algunas opciones de fondo ${ }^{3}$.

\section{El debate sobre el uso de la Biblia en la teología moral}

Durante siglos la teología moral, siguiendo una metodología que era corriente en toda la teología, tomó la Biblia como un depósito de dicta probantia, específicamente, como un reservorio de preceptos, que podían ser extraídos de su contexto original y aplicados en otros contextos sin necesidad de ningún proceso hermenéutico. Si bien, este modo de aproximación a la Sagrada Escritura es muy anterior al Concilio de Trento, en la moral postridentina esta práctica se insertó en un nuevo cuadro conceptual.

Hasta entonces, la visión de la vida moral como camino a la bienaventuranza, y la centralidad de la idea de virtud como esencial en este camino, había equilibrado en alguna medida las tendencias legalistas también presentes en la tradición, garantizando todavía cierto respiro bíblico en este ámbito. Esta concepción comenzó a cambiar en el siglo xIV por influencia del nominalismo, y para el siglo XVII se puede observar claramente cómo en los textos para la formación teológica y pastoral el lugar central no es ocupado ya por los conceptos de felicidad o virtud, sino por el deber. Esta figura de la "moral de la obligación" incidió en un empobrecimiento del recurso a la Biblia, en la cual solo se consideraron de interés para la moral los textos legislativos, principalmente el decálogo. Por este motivo, pasajes de decisiva importancia como las bienaventuranzas o el Sermón de la Montaña, fueron desplazados del ámbito moral y

“Una mayor participación de los moralistas en la redacción del documento habría revestido esta estructura de base [el binomio "llamada-respuesta"] de un conocimiento más completo de la complejidad de esta respuesta", Alberto de Mingo Kaminouchi, "Una teologia morale più «nutrita dalla doctrina della Sacra Scrittura", en Vicenzo Viva y Gabriel Witaszek (coord.), Etica teologica nelle correnti della storia (Vaticano: Lateran University Press, 2011), 22-23 (traducción mía). 
confinados al campo de la espiritualidad, por no contener preceptos específicos ${ }^{4}$.

Ya en el siglo XIX, representantes de la escuela protestante de Tubinga intentaron superar esta limitación dando a la moral un talante más bíblico y evangélico. Este camino fue seguido en el siglo xx por F. Tillmann, quien en su obra «La idea del seguimiento de Cristo» buscó tender el puente entre la teología moral y la Sagrada Escritura, valiéndose de la categoría de ejemplaridad (Cristo-Modelo). Posteriormente, su trabajo sería recogido y profundizado en el ámbito católico por B. Häring, quien lo incorporó en un planteo más amplio de carácter personal-responsorial. Sin embargo, el problema metodológico estaba lejos de su resolución, conservándose una tendencia al positivismo bíblico, a la acumulación de citas, y al confinamiento de la "parte bíblica" en capítulos introductorios, con escasa incidencia en la sistemática y en los contenidos de las obras.

El Concilio Vaticano II, en Dei Verbum llamó a la Sagrada Escritura "el alma de la teología" (n. 24), y el documento sobre la formación de los futuros sacerdotes, Optatam Totius, señaló la necesidad de una teología moral "más inspirada en la Sagrada Escritura", que permitiera mostrar "la grandeza de la vocación de los fieles en Cristo y su obligación de producir frutos en la caridad para la vida del mundo" (n. 16). Pero el entusiasmo inicial suscitado por esta invitación del Concilio y el extendido consenso sobre la necesidad de superar el racionalismo de la moral precedente, tropezaría con las mismas dificultades del pasado.

A esta altura, nadie dudaba de la necesidad de un recurso más amplio y sistemático a la Sagrada Escritura, que permitiera superar

Cf. Servais Pinckaers, Las fuentes de la moral cristiana (Pamplona: Eunsa, 1988), 313s., donde desarrolla un panorama histórico, algo esquemático, que sin embargo ha recogido una amplia aceptación. El mismo ha sido precisado y profundizado por Giuseppe Abbà, Quale impostazione per la filosofia morale? (Roma: LAS, 1995). Para un estudio de este problema en la manualística, y los sucesivos intentos de "relanzamiento" de la Sagrada Escritura, Vincenzo Viva, "La scrittura nella manualistica teologico-morale. Tappe storiche e nodi problematici", Studia Moralia 46/1 (2003): 11-38. 
el legalismo y el uso ingenuo a los textos bíblicos como indicaciones normativas directas. Pero la vieja tensión entre razón y fe se repropuso en el enfrentamiento entre quienes sostenían que la Biblia solo puede brindar un nuevo horizonte de comprensión y nuevas motivaciones, correspondiendo a la razón el trabajo de elaboración de las normas éticas ("moral autónoma"), y quienes abogaban por la primacía de la reflexión bíblica, enfatizando tanto la dificultad de la razón caída para alcanzar el conocimiento moral, como la originalidad de la moral bíblica y evangélica ("ética de la fe") ${ }^{5}$.

En particular, B. Schüller sostuvo que la Sagrada Escritura es fuente de contenidos morales solo en sentido parenético, es decir, en cuanto a exhortaciones a cumplir el bien y evitar el mal ya conocidos. El decálogo mismo, a juicio de este autor, no deja de ser una parénesis, ya que prohibiciones como la de no matar (= no cometer homicidio), requieren siempre de un desarrollo normativo que permita conocer a qué acciones se alude en concreto (en el ejemplo, qué acciones pueden describirse como "homicidio"). ${ }^{6}$ Entre los mayores críticos de esta postura se encuentra S. Pinckaers, quien todavía sostenía la posibilidad de encontrar en la Biblia normas de acción, sobre todo en el sentido de normas absolutas ${ }^{7}$.

Como sucedió con otros debates de la teología moral posconciliar, este fue perdiendo fuerza, no a causa de un esclarecimiento del problema, sino por la razón contraria: la insuperable confusión que fue trabando la discusión hasta generar un comprensible cansancio. Pero la dificultad de fondo subsiste, y es fuente de importantes desacuerdos en los temas normativos concretos. Quisiera presentar un breve ejemplo de esto último: el debate en torno a la homosexualidad. Compagnoni y otros (coord.), Nuevo Diccionario de Teología Moral (Madrid: Paulinas, 1992), 124-127.

6 Bruno Schüller, La fondazione dei giudizi morali (Milano: San Paolo, 1997), 24-27.

7 Cf. Servais Pinckaers, Ce qu'on ne peut jamais faire. La question des actes intrinsèquement mauvais. Histoire et discussion (Fribourg - Paris: Cerf, 1987). 


\section{Biblia y homosexualidad}

Tradicionalmente, la condena de la homosexualidad se fundó en textos que parecían referirse de modo explícito a este tema: el episodio de Sodoma y Gomorra (Gn 19,1-6), la legislación del Levítico (Lv 18,22; 20,13), y diversos textos de San Pablo (ej. 1 Cor 6, 9-10). La exégesis contemporánea, sin embargo, pone en duda la aplicabilidad de esos textos al problema mencionado ${ }^{8}$.

El episodio de Sodoma y Gomorra, por ejemplo, parece subrayar en primer lugar el carácter sagrado del deber de hospitalidad, que impedía a Lot abandonar sus huéspedes a merced de la multitud. En todo caso, si los vecinos de Sodoma pretendían abusar de ellos (en lo que no todos los exegetas concuerdan) se trataría de una cuestión de violencia sexual. Por consiguiente, la homosexualidad no parece ser aludida de modo directo.

En los textos citados del Levítico, se condena explícitamente al varón que tiene relaciones con otro "como si fuera una mujer". Sin embargo, se discute el sentido de esa conducta. Parece tratarse de una manera de prostitución ritual ligada a los cultos idolátricos. La condena podría estar motivada en esto último, careciendo por lo tanto de relación con el fenómeno de la homosexualidad como tal.

Finalmente, los textos paulinos presentan problemas terminológicos difíciles de resolver. ¿Quiénes son, por ejemplo, los "afeminados" (arsenokoitai, 1 Cor 6, 9 y 1 Tim 1,10)? ¿Cuál es la relación entre estos casos y la homosexualidad? Por otro lado, San Pablo solo podía conocer el fenómeno que es producto de la disipación moral, y no el que surge de una determinada estructuración de la personalidad psico-sexual. ¿Pueden identificarse sin más estos dos supuestos? Sexual Ethics (London: Continuum, 2008), 273-277; Todd Salzmann y Michael Lawler, The sexual person. Toward a renewed Catholic Anthropology (Washington D.C.: Georgetown University Press, 2008), 215-226. En contra, cf. Innocent Himbaza, Adrian Schenker y Jean-Baptiste Edart, Consideraciones sobre la homosexualidad en la Biblia (Madrid: Palabra, 2008), 145-149. 
Ahora bien, el hecho de que la enseñanza tradicional sobre la ilicitud de la homosexualidad no pueda apoyarse claramente en un texto determinado, ha llevado a diversos especialistas a sostener que la Biblia no da orientaciones relevantes sobre el tema. En el fondo, unos y otros comparten la misma aproximación legalista a la Escritura: si no es posible encontrar una condenación explícita, solo resta afirmar que la homosexualidad entra en el campo de lo "permitido".

En este punto, la Carta sobre el cuidado pastoral de las personas homosexuales (1987), refleja un momento de transición. En este documento se emplea el método tradicional de fundamentación normativa sobre la base de textos aislados, precisamente los que más arriba hemos recordado. Pero al mismo tiempo, y en primer lugar, se recurre a un concepto diferente: la teología de la creación contenida en la revelación bíblica, y su correspondiente visión del hombre y del mundo. Ello explicaría a mi juicio por qué, considerada en la perspectiva "canónica", es decir, de la Sagrada Escritura tomada en su conjunto, de una manera u otra la conducta homosexual aparece siempre bajo una luz negativa, aunque ningún pasaje aisladamente y por sí mismo permita arribar a un juicio concluyente. Si bien de aquí no se puede inferir ninguna conclusión directa, nos encontramos ante un dato insoslayable para la reflexión moral.

Pero esta novedad que despunta en la Carta necesitaba un desarrollo y una justificación cuidadosa. Este es, precisamente, el mérito del documento que analizamos.

\section{3. iSe pueden encontrar en la Biblia "normas e indicaciones"?}

El título de este apartado hace referencia a una afirmación del cardenal Levada en el prólogo del documento: "Los cristianos están convencidos de que en la Biblia se pueden encontrar indicaciones y normas para obrar rectamente y alcanzar la vida plena" (prólogo ByM). En el contexto de las discusiones que se habían suscitado 
en las décadas anteriores en torno a la pretensión de encontrar normas morales en la Biblia, no solo en sentido formal (parénesis) sino sobre todo en sentido material-operativo, la frase resulta a primera vista polémica.

Sin embargo, a continuación esta primera afirmación es cualificada fuertemente:

1. En primer lugar, se aclara que "en la perspectiva bíblica, un discurso sobre normas morales no se puede reducir a ellas mismas tomadas en forma aislada, sino que siempre debe estar inserto en el contexto de la visión bíblica de la existencia humana" (Prólogo, MyB). Todo "hallazgo" normativo debe ser fruto de un procedimiento hermenéutico.

2. Más aún, se reconoce que la Biblia no ofrece "soluciones prefabricadas", sino que "presenta criterios cuya aplicación ayuda a encontrar soluciones válidas para el comportamiento humano" (Prólogo, MyB). A la necesidad del trabajo interpretativo (que excluye las "soluciones prefabricadas") se agrega otro elemento: lo que se obtendrá, se reconoce, no son normas sino criterios para formular normas.

3. Finalmente se agrega que "se trata de mostrar cuáles son los puntos que la revelación bíblica ofrece para ayudarnos, hoy, en el delicado proceso de un justo discernimiento moral" (Prólogo, MyB). El texto bíblico, según esto, ofrece puntos de partida y orientaciones para el ejercicio del discernimiento moral. Este último requiere no solo la contextualización del texto bíblico, sino también la consideración de la situación real para la cual se piensa una solución normativa, así como otras consideraciones propias de la actividad prudencial.

Pese a la sonora afirmación inicial, comprendemos al final que lo que se pretende no es en realidad encontrar normas en la Biblia, sino a partir (y a la luz de) de la Biblia. 


\section{Los presupuestos y los objetivos del documento}

El hecho de que la teología moral se acerque a la Biblia con la intención de encontrar en ella, o mejor, de elaborar a partir de ella, normas de acción, no significa que estas normas constituyan el fin último de su labor. En efecto, como reza el texto del prólogo de ByM que citamos en el apartado anterior, se trata de "encontrar indicaciones y normas para obrar rectamente y alcanzar la vida plena". Este es el objetivo último de esta búsqueda: "la vida plena", y por lo tanto también ésta será la pregunta que el teólogo y el exegeta deben dirigir en primer lugar al texto bíblico: la pregunta por la felicidad y el sentido de la vida.

Se comprende la significación de este punto de partida cuando se compara esta aproximación al texto inspirado con la que predominó en los siglos anteriores, de corte reductivamente normativo, centrado en la pregunta por los preceptos. Como ya hemos indicado, este modo de acercamiento a la Escritura se focalizaba en los textos legales, dejando de lado otros de decisiva importancia para la moral, como las bienaventuranzas ${ }^{9}$, o el Sermón de la Montaña.

Como ha mostrado S. Pinckaers, este enfoque restringido, producto de una "moral de la obligación" centrada en el concepto de deber, puede ser superado solo cuando el intérprete parte de la pregunta por la felicidad, logrando merced a ello una comprensión más adecuada de la riqueza moral de la Biblia $^{10}$. Y siendo la felicidad una aspiración que compartimos todos los seres humanos por el hecho de ser tales, la respuesta bíblica también tiene un alcance universal. Más aún, la pregunta por la felicidad contiene implícitamente la pregunta por Dios (cf. VS 9) ${ }^{11}$. El presupuesto tácito, que es asumido

Cuya auténtica relevancia para la vida en Cristo fue rescatada por el Catecismo de la Iglesia Católica 1716-1729.

10 Cf. Servais Pinckaers, Las fuentes de la moral cristiana, op. cit., 142-148.

11 VS 9 se refiere explícitamente solo a la pregunta sobre el bien, pero este bien en última instancia se identifica con la felicidad y la vida plena. Cf. ibíd. 
por ByM, es que "Dios es, para todo creyente y para todo hombre, la respuesta última a esta búsqueda de felicidad y de sentido" (n. 1).

De esta íntima vinculación entre la pregunta moral y la pregunta por Dios se sigue entonces un segundo presupuesto, el del significado universal de la Biblia, que por el mismo hecho de ser la Palabra de Dios constituye un espacio de diálogo incluso con los no creyentes: "La Sagrada Escritura como unidad, es decir, comprendiendo los dos testamentos, es un lugar válido y útil de diálogo con el hombre contemporáneo sobre cuestiones que atañen a la moral" (ByM n. 1).

Esta universalidad dinámica (que se va descubriendo progresivamente a través del diálogo) no comporta la negación de la "especificidad y originalidad" de la revelación en el plano ético frente a otras morales naturales o religiosas. Por el contrario, afirmar esa especificidad constituye el primer objetivo del documento. Sin embargo, la misma no se traduce de un modo directo en normas de acción. Sostener lo contrario equivaldría a poner en tela de juicio la autonomía de la razón moral y la comunicabilidad de la moral cristiana.

De acuerdo al segundo objetivo, de carácter práctico, ByM se propone la elaboración de criterios metodológicos que "faciliten el camino" hacia respuestas potencialmente universales a los problemas morales concretos. Veremos más adelante la caracterización de dicha originalidad en su aspecto positivo.

\section{La "moral revelada" como concepto clave}

ByM introduce como concepto clave el de "moral revelada" (n. 4), aun reconociendo que el mismo "quizás no es habitual". Esta opción, sin embargo, se presta a interpretaciones equivocadas.

En primer lugar, la expresión "moral revelada" puede evocar problemáticas asociaciones con una visión heterónoma de la moral. Si hace referencia a contenidos revelados, żquiere decir que los mismos están por principio fuera del alcance de la razón natural? Y si es así, ¿̇cómo puede el sujeto moral reconocerlos como obligatorios? 
¿Solo por la "obediencia de la fe"? Y en ese caso, ¿̇podría seguirse afirmando su comunicabilidad, la idoneidad de la Sagrada Escritura como ámbito para el diálogo universal?

Por otro lado, esta expresión es más "habitual" que lo que el documento parece suponer. La misma es bien conocida al menos en el mundo de habla inglesa, a partir sobre todo de un artículo de gran resonancia escrito por el teólogo protestante James Gustafson ${ }^{12}$, en el cual este autor contrapone los conceptos de "moral revelada" (revealed morality) y "realidad revelada" (revealed reality). La primera expresión indica la convicción de que en la Biblia es posible encontrar contenidos normativos (preceptos, ideales, analogías) aplicables de modo más o menos directo a otros contextos. La segunda, en cambio, entiende que la función específica de la Sagrada Escritura es la de arrojar una luz original sobre la realidad, en primer lugar, sobre la acción salvífica de Dios, que motiva la respuesta del hombre, y constituye el punto de partida del trabajo ético-normativo ${ }^{13}$.

El significado corriente de la expresión "moral revelada" parece ser, entonces, distinto al que el documento le atribuye. ¿̇Se ha asumido este concepto con plena conciencia de las dificultades interpretativas que se pueden generar, o esta opción ha sido el resultado de un insuficiente asesoramiento? En todo caso, ByM lo refiere "al movimiento de fondo de la Escritura en su totalidad" (n. 4), es decir, a la dinámica de la revelación, no a su contenido ${ }^{14}$. Lo primero y fundamental es

James Gustafson, "The Place of Scripture in Christian Ethics: A Methodological Study", en Charles Curran y Richard McCormick (coord.), Readings in Moral Theology, vol. 4: The Use of Scripture in Moral Theology (New York: Paulist Press, 1984), 151-177. Esta distinción se inspira en Richard Niehbur, The Meaning of Revelation (New York: Macmillan, 1941).

13 Así lo reconocía R. Niehbur: "Lo que la Biblia da a conocer, entonces, no es una moral, sino una realidad, una presencia viviente a la cual el hombre responde. Para cuestiones de moral, su autoridad es «educacional», dando a los hombres conocimiento de sí mismos y conocimiento de Dios, en cuya luz ellos interpretan como sus responsabilidades y actúan: su autoridad es «corroborativa», proveyendo un criterio de validación que ayuda a la comunidad cristiana a ver sus propias perversidades y a verificar sus verdaderos propósitos". Citado por James Gustafson, "The Changing Use of the Bible in Christian Ethics", op. cit., 143.

14 Cf. DV 2. Sobre la repercusión de este desarrollo del concepto de revelación en la teología moral, Alberto de Mingo Kaminouchi, op. cit., 15-17. 
la iniciativa de Dios, que en la revelación se comunica a sí mismo, el misterio de su ser y de su voluntad. La experiencia de este don es lo que permite al hombre, en un segundo momento, la respuesta moral ${ }^{15}$. Es más, la misma Ley es don, en cuanto "camino" por el cual el hombre se incorpora en la dinámica de la Alianza.

Como puede apreciarse, esta explicitación del significado de la "moral revelada" no se presta a acusaciones precipitadas de heteronomía o positivismo escriturístico. Sin embargo, tampoco aparece claro todavía de qué modo se concilia aquella con la autonomía de la razón moral, como puede notarse en el siguiente pasaje:

Mientras se reduce la moral a un código de comportamientos individuales y colectivos, a un conjunto de virtudes que se deben practicar o también a los imperativos de una ley natural que se entiende como universal, no se puede percibir suficientemente toda la especificidad, la bondad y la actualidad permanente de la moral bíblica. (n.4.1).

La frase citada genera perplejidad. Aquí se busca ilustrar la originalidad de la moral revelada a través de un contraste con visiones reductivas de la moral: el legalismo (la moral como código de normas o conjunto de virtudes-deberes) y el racionalismo (concepción a-histórica de la ley natural). Pero el término de comparación, ¿̇no debería ser la moral natural en sí misma, y no en sus versiones "reductivas"? ¿̇ toda moral natural es constitutivamente reductiva? Se comete aquí el error de confrontar conceptos heterogéneos: la "moral revelada" es tomada como concepto ideal, no histórico (es decir, prescinde de los límites humanos en la comprensión de la misma), mientras que la moral natural es tomada como concepto histórico (sus figuras defectuosas), no como ideal ${ }^{16}$.

Por otro lado, llama la atención que se ponga en la misma línea normas, virtudes, e imperativos de la ley natural. La ética de la vir-

15 De este modo, la relación entre la iniciativa divina y la respuesta humana constituye el "dato más fundamental", estructurante de todo el documento: los tres grandes dones de Dios (la creación, la Alianza antigua, la Alianza nueva) y el perdón. Cf. Klemens Stock, op. cit., 6-13.

16 Cf. Bruno Schüller, "Zur theologischen Diskussion über die lex naturalis", Theologie und Philosophie 4 (1966): 495. 
tud, como camino de abordaje de la moral bíblica, hubiera merecido mayor reconocimiento. A su vez, la referencia a "una ley natural que se tiene por universal", ¿̇está sugiriendo que solo la "moral revelada" es capaz de una verdadera universalidad?

\section{Moral revelada y moral natural}

Las preguntas precedentes nos muestran la necesidad de definir de un modo más adecuado la relación entre moral revelada y moral natural, y esclarecer con una caracterización positiva la afirmación de la originalidad de la primera. Al respecto, se cita más adelante al teólogo J. Ratzinger:

La originalidad de la Sagrada Escritura en el ámbito moral no consiste en la exclusividad de los contenidos propuestos, sino en la purificación, en el discernimiento y en la maduración de todo lo que la cultura ambiente proponía. Se trata de un discernimiento crítico de lo que es verdaderamente humano porque nos asimila a Dios, y su purificación de todo lo que es deshumanizante y de "su inserción en un nuevo contexto de sentido, que es el de la Alianza" (n. 95).

Este texto aporta lo que faltaba en la explicación del concepto de "moral revelada": la conciliación de la misma con la autonomía de la razón moral. La revelación no opera una sustitución sino una purificación de la razón natural.

Por otro lado, el texto de ByM hubiera alcanzado un mejor equilibrio reconociendo una verdad complementaria: así como la moral revelada ayuda a discernir lo que es "verdaderamente humano", la moral natural es indispensable para conocer cuál es en la Biblia la moral efectivamente revelada. La relación entre moral natural y revelada no debe entenderse en modo unidireccional, como ByM parece proponer, sino en términos de un círculo hermenéutico, en que ambas se esclarecen e interpretan mutuamente ${ }^{17}$.

Teniendo en cuenta que tanto la moral revelada como la moral natural se encuentran situadas en contextos históricos y culturales concretos, esta interacción debe adquirir la forma de lo que H.-G. Gadamer denominaba la "fusión de horizontes". Cf. Alberto de Mingo Kaminouchi, op. cit., 25-26. 
De todos modos, queda clara la idea de continuidad entre ambas, a través de la integración de las verdades accesibles a la razón natural en el contexto dialógico de la Alianza. Es claro lo que esto último significa en lo que respecta a las motivaciones y al horizonte de comprensión, quedando abierta la cuestión de cómo caracterizar esa continuidad en el nivel de los contenidos normativos.

\section{Ley y leyes}

Si bien la originalidad de la moral bíblica no debe buscarse en el nivel de los contenidos, ByM se propone dar criterios para elaborar enunciados normativos. En efecto, el documento distingue entre Ley en sentido teológico, como "camino de vida", y las leyes, como la práctica de determinaciones precisas (n.24). La primera refleja la respuesta del hombre a Dios "en su globalidad teológica inmutable", mientras que las segundas son "su expresión plural, detallada y eventualmente adaptable a las circunstancias" (n.24). ByM se ocupa también de estas últimas.

La distinción entre Ley y leyes en un sentido teológico es clara, pero la interpretación que hace el documento de esta diferencia en el plano moral llama la atención. Durante las décadas posteriores al Concilio se discutió ásperamente sobre la existencia de "absolutos morales", en el sentido de normas de acción negativas o prohibitivas que pudieran valer semper et pro semper, tema central de la encíclica Veritatis splendor. ByM parece sugerir una identificación entre el nivel de las normas de acción y el ámbito de los contenidos adaptables a las circunstancias, ("las leyes", en plural). Y lo hace precisamente antes de estudiar las normas del decálogo, el texto en que la mencionada encíclica fundamenta su afirmación sobre la existencia de comportamientos intrínsecamente malos (VS 13).

De esta manera, la distinción entre Ley y leyes según ByM parece volver a proponer la polémica distinción, frecuente en décadas pasadas, entre nivel trascendental y categorial, o entre normas for- 
males y materiales, atribuyendo a los absolutos morales un sentido exclusivamente trascendental y formal.

\section{Alianza y modelos éticos}

De todos modos, esta comprensión teológica de la ley corrobora lo que observara en su momento Ch. Curran: "la renovación bíblica, con su énfasis en la alianza y el amor de Dios se orienta en cierto sentido en contra del modelo deontológico"18. Este modelo, que imposta la moral bíblica en términos de deberes y es por lejos el más popular en la vida de los católicos, ocupa en ByM un lugar "sin ser secundario, segundo". Ello, sin embargo, no induce a este documento a inclinarse a favor de algún "ideal" bíblico único que polarice el conjunto de la vida moral, conforme al modelo teleológico (como lo fuera, por ejemplo, la noción de Reino de Dios en el protestantismo liberal). Sin rechazar los modelos precedentes, ByM vincula el concepto de alianza al esquema de don y respuesta, lo que equivale a asignar la primacía a una visión centrada en la responsabilidad ética, en la cual la ley adquiere el significado de un compromiso personal y colectivo con Dios.

A la luz de este último modelo, es posible esclarecer más adecuadamente el significado de la inserción de la ley en el contexto de sentido de la Alianza. La iniciativa divina encuentra al hombre sumergido en la conflictualidad de la historia, que ofusca la percepción moral y paraliza la potencia de su libertad en su decisión por el bien. En este contexto, la relación personal con el Dios vivo constituye un "camino de vida" en cuanto permite al creyente abrirse a nuevas percepciones morales y, con ellas, a nuevas alternativas de acción ${ }^{19}$. En esto radica el carácter progresivo de la moral bíblica, que se convierte así en una analogía que ilumina el dinamismo de 
toda la vida moral. En consecuencia, conceptos como crecimiento, desarrollo, creatividad, interioridad, y totalidad personal pasan a ocupar un primer plano en la reflexión ética ${ }^{20}$.

\section{Interpretación axiológica del decálogo}

Un claro ejemplo de lo dicho es la presentación que ByM hace del decálogo. Se trata de un texto legislativo de especial importancia ${ }^{21}$, pero en comparación con el ideal cristiano presenta notorios límites, debido a su exterioridad, su alcance esencialmente comunitario y su formulación frecuentemente negativa (n. 25-26). Resulta sugestivo el hecho de que el documento se limite a interpretar el carácter negativo de varios de los preceptos del decálogo como mera expresión de un mínimo moral, una "ética inicial aunque potencialmente muy rica", sin hacerse eco de la importancia radical que VS atribuye a estas normas negativas como fundamento de la objetividad moral, en cuanto protegen el bien de la persona frente a comportamientos que lo vulneran (cf. VS 13).

Pero esta primera lectura del decálogo, de carácter literal, da pie para proponer otra forma de interpretación, una lectura axiológica, capaz de superar los "inconvenientes" mencionados. Mientras que la prohibición se reduce a ciertos comportamientos que se deben evitar, y también el precepto positivo puede satisfacerse con actos que no superan el mínimo, el compromiso con el valor es "una cantera siempre abierta", de fecundidad inagotable.

Así, cada mandamiento es vinculado con un determinado valor: el primero se refiere al culto del único Absoluto; el segundo al respeto de su presencia y misión (su "Nombre"); el tercero, a la dimensión sagrada del tiempo; etc. (cf. n. 30). Esta traducción axiológica del decálogo no parece que deba tomarse en un sentido limitativo: por 
ejemplo, no aparece en modo explícito el valor de la veracidad ${ }^{22}$. Por otro lado, la definición de algunos valores desconcierta: el derecho sobre los bienes materiales curiosamente no forma parte del séptimo mandamiento (no robarás), sino solo del décimo. Pero lo que es más importante señalar es el hecho de que ByM ha hecho una opción, al parecer inadvertida, por un modo de interpretar los mandamientos: considerándolos como parénesis, es decir, exhortación, un llamado a realizar valores pero que, como tal, se mantiene en un nivel formal, sin indicar todavía contenidos materiales.

La "adaptabilidad" a la que ByM hace referencia no se encuentra en estas "leyes" de carácter más formal, ni tampoco en las conclusiones de la lectura axiológica, que también son formales (cf. n.30-31). Dicha adaptabilidad debe predicarse, por lo tanto, solo de aquellas "leyes" que constituyen normas materiales, de acción, sin que el documento reconozca la existencia en este nivel de normas absolutas y por lo tanto "no adaptables".

\section{Derecho apodíctico y derecho casuístico}

En este punto, el documento depara otra sorpresa, al asumir la vieja distinción entre derecho apodíctico y derecho casuístico (n. 151), con la que en su momento se quiso confirmar el carácter distintivo y único de la moral revelada, la cual estaría contenida exclusivamente en las leyes apodícticas ${ }^{23}$. Según ByM, estas últimas (Ex 22,20 - 23,9) están dotadas de fundamento teológico (la cercanía del Señor con las categorías más pobres), y por lo tanto, tendrían un valor que trasciende las culturas. Las leyes casuísticas, por el contrario, estarían más ligadas a la cultura ambiental, y son con frecuencia muy similares a las prescripciones de diversos códigos del Cercano Oriente antiguo (por ejemplo, la liberación periódica de esclavos, Ex 21,2-11). 
Pero como se ve en el ejemplo citado, la benevolencia hacia el esclavo, la norma casuística puede tener también un contenido transcultural. El hecho de que revista una formulación hipotética ("si sucediera $X^{\prime \prime}$; "en caso de $Y^{\prime \prime}$; etc.) no indica un menor valor moral respecto de las leyes apodícticas. Las normas casuísticas cumplen una función indispensable concretando el contenido que en las normas apodícticas se encuentra sin explicitar ${ }^{24}$. No se analiza además la relación entre ambos niveles, y nuevamente las normas de acción son situadas en el campo de lo contextual y adaptable. Por otro lado, la "casuística" aparece siempre en el documento bajo una luz negativa, identificada sin más con el legalismo, lo cual también dificulta su correcta valoración.

En conclusión, la "moral revelada" es situada en el campo del derecho apodíctico, que a su vez parece identificarse con las "leyes" de carácter más formal, sin que quede claro el vínculo con la casuística y con las normas concretas. La separación entre ambos planos, buscada o no, parece ser un patrón que se repite a lo largo del documento.

\section{Los criterios fundamentales}

Tener presente este análisis del marco conceptual, es importante a la hora de estudiar los criterios de interpretación. Para la idea de "moral revelada" propuesta por el documento, la prioridad corresponde al indicativo, es decir, a la descripción del obrar de Dios que funda el imperativo moral. Por consiguiente, el gran desafío es cómo pasar del indicativo al imperativo sin caer en formas más o menos disimuladas de intuicionismo, en otras palabras, cómo formular un método crítico-racional que permita vincular ambas dimensiones.

Aquí reside el mayor mérito del documento, que luego de descartar toda aproximación ingenua al texto bíblico, insiste correctamente

Cf. Bruno Schüller, La fondazione dei giudizi morali, op. cit., 33-36. 
sobre la necesidad de una hermenéutica cuidadosa, y brinda valiosas orientaciones para realizar esta tarea. En primer lugar, se mencionan dos criterios fundamentales: (1) la visión bíblica del ser humano; y (2) la conformidad con el ejemplo de Jesús.

El primer criterio se refiere a la relación entre las conductas y la visión integral del ser humano en cuanto imagen de su Creador, llamado individual y colectivamente a la comunión con Dios y con sus semejantes. Se lo ejemplifica a través de la lectura axiológica del decálogo, a la que ya hicimos referencia, a fin de mostrar cómo las ideas de creación y de alianza pueden expandir nuestra comprensión de los valores, en este caso, el valor de la vida y el de la pareja conyugal (nn. 96-99).

El segundo criterio se refiere al tema tradicional de la imitación de Jesús, modelo de conformidad con la voluntad de Dios. Esto no debe entenderse en el sentido de un "ideal más o menos inaccesible" o una vaga inspiración, sino como verdaderos "imperativos morales", que Jesús no solo enseña, sino que encarna en su vida (n. 100; 102). Estos imperativos, sin embargo, son definidos a continuación como "un horizonte de fondo que conduce a los discípulos a buscar y encontrar modos semejantes para ajustar la propia acción a los valores y la visión de fondo del evangelio" (n. 102) ${ }^{25}$. Como se puede apreciar, prevalece también aquí la cautela frente a la posibilidad de lecturas demasiado literales. En esta clave cristológica, ByM lee las bienaventuranzas y la exhortación a la "justicia superior" en el Sermón de la Montaña, y sitúa acertadamente el seguimiento de Cristo en la asimilación de las actitudes de fondo de su existencia, especialmente, su espíritu filial. el ejercicio de la "imaginación analógica". Cf. Aristide Fumagalli, "Sacra Scrittura e agire morale. Una proposta teoretica", Studia Moralia 47/4 (2009): 88-91. Se superan así los límites de una ejemplaridad paradigmática (exterior) en pos de una moral hypo-digmática, en la que el ejemplo (hypodeigma, Jn 13,15) de Jesús, su modo de vivir su condición filial, se convierte en raíz de la vida del creyente, como la vid nutre a los sarmientos (Jn 15). Cf. Adrzej S. Wodka, "Bibbia e morale: rilievi di un biblista", en Vicenzo Viva - Gabriel Witaszek (coord.), Etica teologica nelle correnti della storia, (Vaticano: Lateran University Press, 2011), 36. 


\section{Criterios específicos}

A continuación, ByM enuncia una serie de criterios más específicos, cuyo elenco no posee carácter exhaustivo (n. 104ss.).

\begin{tabular}{|c|c|c|c|}
\hline Criterio & Definición & $\begin{array}{c}\text { Palabra } \\
\text { Clave }\end{array}$ & Algunos Temas \\
\hline Convergencia & $\begin{array}{c}\text { Apertura a la } \\
\text { ley natural }\end{array}$ & Sabiduría & $\begin{array}{c}\text { Corresponsabilidad } \\
\text { (derechos humanos, } \\
\text { paz, técnica, etc.) }\end{array}$ \\
\hline Contraposición & $\begin{array}{c}\text { Rechazo de } \\
\text { contra-valores }\end{array}$ & Fe & $\begin{array}{c}\text { Contra idolatrías } \\
\text { (tendencias totalitarias) }\end{array}$ \\
\hline $\begin{array}{c}\text { Progresión } \\
\text { Comunitaria }\end{array}$ & $\begin{array}{c}\text { Afinación } \\
\text { gradual de la } \\
\text { conciencia }\end{array}$ & $\begin{array}{c}\text { Justicia } \\
\text { (Voluntad } \\
\text { individualismo }\end{array}$ & $\begin{array}{c}\text { Dios) } \\
\text { Progresividad (perdón, }\end{array}$ \\
\hline fraterno & $\begin{array}{c}\text { Amor y comulto espiritual) } \\
\text { (familia, iglesia, } \\
\text { sociedad civil, etc.) }\end{array}$ \\
\hline Finalidad & $\begin{array}{c}\text { Horizonte } \\
\text { escatológico }\end{array}$ & Esperanza & $\begin{array}{c}\text { El futuro escatológico, } \\
\text { impulso al } \\
\text { compromiso con } \\
\text { el presente }\end{array}$ \\
\hline Discernimiento & $\begin{array}{c}\text { Aproximación } \\
\text { crítica }\end{array}$ & Prudencia & $\begin{array}{c}\text { Lectura personal y } \\
\text { comunitaria de la biblia }\end{array}$ \\
\hline
\end{tabular}

Si bien como dijimos esta lista es solo "representativa", los criterios están muy bien elegidos. El criterio de convergencia, indica el nivel de la moral natural. Los criterios de contraposición, comunidad y finalidad, están referidos a las virtudes teologales, como surge de las "palabras-clave" asignadas a ellos: fe, caridad y esperanza, 
respectivamente (n. 104). El criterio de progresión se vincula a la justicia en sentido bíblico, la búsqueda de la voluntad de Dios, reconociendo su carácter gradual e histórico. Se lo puede considerar como la proyección moral de la vida teologal. Finalmente, el criterio del discernimiento permite elaborar, a partir de los criterios precedentes, los juicios prácticos relativos a las normas de acción y a los comportamientos concretos. Si consideramos, además, el dinamismo implícito en este elenco de criterios, se verá cómo se comienza asumiendo de un modo crítico la moral natural, para integrarla en el movimiento de la vida teologal, desde las actitudes de fondo hasta el obrar concreto.

Refiriéndonos a los criterios en particular, se observa cómo el criterio de la convergencia casi no se vincula con la expresión "ley natural" (que considera "externa" a la Sagrada Escritura, cf. n. 159) sino con la idea de una "sabiduría natural", entendida como "valor potencialmente universal" (n. 105, ${ }^{26}$. Esto tal vez signifique que en la visión de ByM aquella solo puede adquirir un carácter actualmente universal a través de un ejercicio de "diálogo" y "búsqueda común" con otras religiones y filosofías. En lugar de ley natural se prefiere hablar de "la capacidad de la conciencia humana para distinguir lo que se debería hacer y lo que no" (n. 109) lo que hace posible una convergencia "en muchos puntos" (n. 105). En su conjunto, este tipo de afirmaciones esparcidas en el documento parecen indicar una actitud más cautelosa frente a la ley natural que la expresada hace pocos años en el documento de la Comisión Teológica Internacional sobre este tema ${ }^{27}$. De hecho, la idea de que el decálogo es "una expresión privilegiada y siempre válida de la ley natural" no ha sido recogida ${ }^{28}$.

El criterio de contraposición muestra cómo la Biblia no asume sin más las normas y costumbres de su ambiente, sino que se opone a

27 Comisión Teológica Internacional, En búsqueda de una ética universal: nueva mirada sobre la ley natural (Marzo 27, 2009), en especial, nn.22-25. www.vatican.va/roman_curia/congregations/cfaith/ cti_documents/rc_con_cfaith_doc_20090520_legge-naturale_sp.html. 
muchas de ellas. Se recurre como ilustración al pecado de idolatría no solo en su versión religiosa, sino también política (divinización del poder del Estado) y existencial (la idolatría de sí mismo). En este punto, se incorpora un elemento silenciado hasta ese momento: la existencia de "una serie de acciones" que constituyen "lo que no se debe hacer" (n. 112.1): el aborto, la eutanasia, la "ilimitada" experimentación genética y las uniones homosexuales, entre otras conductas, son calificadas, sin mayores explicaciones y de un modo indiscriminado, como fruto de la reivindicación de "un derecho a la libertad más total" (n. 117.2). Esta repentina introducción de los "absolutos morales" resulta algo inconsistente, por los motivos que señalé anteriormente. Por otro lado, hubiera sido aconsejable hablar, con mayor amplitud, de un criterio de "purificación" o mejor, de "integración", para indicar que la fe no se limita a un rol negativo (contraponerse a determinados antivalores de la cultura) sino que asume y lleva a plenitud los aspectos auténticamente humanos.

Reviste gran interés la introducción del criterio de progresividad. "Así como la revelación, también la moral bíblica tiene un carácter gradual e histórico" (n. 121). El documento reconoce que las posiciones morales evolucionan, tanto en el interior de la Biblia como "en la historia posterior" (n. 158), y no solo por un cambio de circunstancias, sino ante todo por un "refinamiento" de la conciencia moral. El paso del Talión al perdón sin límites, de la poligamia a la fidelidad conyugal, del culto antiguo al culto espiritual, y su potencial para la elaboración de "estrategias para avanzar paso a paso por el camino de la perfección evangélica" (n. 125) ${ }^{29}$, permiten pensar soluciones pastorales nuevas y creativas "valorando bien el punto del camino al que las personas o grupos han llegado" (n. 125.3). Aquí ya no se trata de la (a veces imposible) tarea de distinguir el núcleo permanente de la norma de su revestimiento contingente e histórico, 
sino de encuadrarla en el "vigoroso dinamismo de conjunto" 30 que atraviesa la Biblia y orienta todo hacia el amor pascual de Cristo.

El criterio comunitario se refiere a la importancia y la fuerza formativa de la pertenencia a una comunidad, en especial la comunidad cristiana, que es el Cuerpo de Cristo y está animada por la fuerza del Espíritu Santo (n. 129). Este criterio permite, por un lado, rectificar "la tendencia a relegar las decisiones morales a la sola esfera subjetiva individual" (cf. Prólogo). Pero no se trata solo de superar el egoísmo individual sino también el colectivo: el amor cristiano es esencialmente inclusivo, respecto de los miembros de la comunidad, los marginados y los que están afuera (nn. 131-133). Quizás hubiera sido oportuno señalar, en homenaje a la preocupación moderna por la libertad del individuo frente a la comunidad, el potencial del concepto bíblico de Alianza como reconocimiento recíproco para la afirmación de la justa autonomía personal.

El criterio de finalidad muestra la repercusión decisiva que tiene la esperanza en la vida eterna, fundada en la fe en la resurrección, en el ámbito de las motivaciones y los comportamientos (n. 135). Esta perspectiva escatológica se anticipa progresivamente en la historia (n. 144), haciendo posible el "absoluto radicalismo" en el seguimiento de Cristo, verificado de modo ejemplar en los mártires (n. 139). Esta referencia a los mártires en ByM difiere visiblemente de VS 90, que interpreta el martirio en clave de obediencia a la Ley de Dios ${ }^{31}$. La esperanza cristiana dirigida al futuro salva al hombre del encierro en el presente (n. 147), y a su vez lo impulsa a comprometerse con él (n. 148).

Resta comentar el sexto y último criterio, el del discernimiento, que por su especial importancia para el objetivo del documento, merece un tratamiento particular.

30 Pontificia Comisión Bíblica, La interpretación de la Biblia en la Iglesia (Ciudad del Vaticano: Libreria Editrice Vaticana, 1993), III.D.3. Cf. Aristide Fumagalli, op. cit., 84.

31 John O'Keefe, "No Place for Failure? Augustinian Reflections on Veritatis Splendor", en Michael Allsopp y John O'Keefe (coord.), "Veritatis Splendor". American Responses (Kansas City: Sheed \& Ward, 1995), 16-37. 


\section{El criterio del discernimiento}

Como vemos, ByM propone un método de interpretación moral de los textos bíblicos que por su amplitud y complejidad resulta muy distante de las aproximaciones ingenuas del pasado. Es comprensible entonces que la exposición de los criterios de interpretación bíblica desemboque en un tratamiento pormenorizado del criterio de discernimiento, abarcando tres niveles: el literario (la lectura crítica que hace el exegeta de los textos); el espiritual-comunitario y el espiritualpersonal. En todos los casos, un "sano discernimiento" supone sentido de las proporciones, capacidad para distinguir entre lo que tiene valor universal y lo contingente, atención a las consecuencias, etc.

En el discernimiento del valor normativo de un texto, deberá prestarse atención al contexto literario, a la existencia o no de un fundamento teológico, al trasfondo cultural, a la continuidad del tema en toda la Escritura (reveladora de su función estructurante), y el lugar que ocupa en el proceso de afinación de la conciencia moral (criterio de progresividad).

A continuación ByM traspone el campo exegético para referirse a la praxis del discernimiento moral, en primer lugar, en cuanto discernimiento de la comunidad eclesial. El análisis de Hch 15,1-35, sobre la situación de los creyentes venidos de la gentilidad respecto de la Ley de Moisés, permite señalar algunas pautas del discernimiento comunitario. En primer lugar, el procedimiento sinodal, que involucra a toda la Iglesia, y permite un auténtico ejercicio de corresponsabilidad. En segundo término, el esfuerzo de "distinguir lo urgente (los valores que se deben salvaguardar) y lo posible (la posibilidad de que una de las partes en causa sea absorbida por la otra)" (n. 152). El modo de expresión no es del todo claro, pero sí lo es el sentido: los apóstoles llegaron a una solución de compromiso, reduciendo al mínimo las cargas para unos y procurando evitar el escándalo de los otros, como modo de preservar la unidad eclesial. Estamos ante una búsqueda de equilibrio propio de las decisiones 
prudenciales. Además, es un discernimiento propiamente espiritual, efectuado en un contexto de oración, y cuyo resultado es dirigido más a la conciencia moral que a la mera obediencia de los destinatarios. El lector podrá apreciar sin necesidad de mayores comentarios el contraste con ciertos procedimientos decisionales en la Iglesia actual.

Por último, se hace referencia al discernimiento a cargo de la conciencia personal (el "corazón" en el lenguaje bíblico, centro de la decisión moral), acertadamente calificado como el más importante de los tres mencionados (n. 153). De los ejemplos que se aportan, es el primero de todos el que parece más claramente pertinente. Se trata del texto paulino de 1 Cor 8,1-11,1, referido a las carnes inmoladas a los ídolos. En este caso, Pablo no se conforma con afirmar el fundamento teológico, a saber, la vacuidad de los ídolos y la soberana libertad del creyente, sino que introduce en su consideración un principio prudencial: la delicadeza de la caridad, que tiene en cuenta a los hermanos de "conciencia débil" procurando evitarles el escándalo. Es una oportuna ilustración de cómo la prudencia no se limita a aplicar deductivamente principios, sino que pondera consideraciones diversas, analizando su valor relativo, sus mutuas correspondencias y sus efectos previsibles.

\section{Teología moral y Biblia: una circularidad hermenéutica}

Por último, quisiera retomar lo dicho más arriba acerca de la circularidad entre moral revelada y moral natural. Este planteo permite superar una cierta unilateralidad de ByM, que enfatiza la función de purificación que ejercita la moral revelada sobre la sabiduría natural, pero casi no se refiere al aspecto complementario de cómo esta última contribuye a discernir qué es lo auténticamente revelado, en lo cual tal vez influya la cautela de ByM frente a la ley natural que hemos indicado anteriormente ${ }^{32}$. del fenómeno ético respecto de la revelación, cf. Martin Mckeever, "Il binomio Bibbia-morale: un nodo da sciogliere", en Vicenzo Viva y Gabriel Witaszek (coord.), Etica teologica nelle correnti della storia, (Vaticano: Lateran University Press, 2011), 66-67. 
Teniendo presente que ambos ethos no se encuentran, por así decirlo, en estado puro, sino encarnados en la conciencia histórica, lo que acabamos de decir significa, en concreto, que la teología moral debe concebirse a sí misma como una ciencia hermenéutica ${ }^{33}$. Es necesario

\begin{abstract}
acercarse al texto bíblico con la previa comprensión que el «texto» de la historia humana de hoy hace posible; con tal comprensión interrogamos la Palabra de Dios y somos interrogados por ella, de modo de retornar al texto de la historia con una más plena y liberada inteligencia de lo humano. De esta manera, el ethos bíblico continúa, a través de los creyentes, a hacerse presente y eficaz en la historia del ethos contemporáneo (...) la consiguiente mejor comprensión de los valores humanos ejercitada al interno de los actuales problemas de comportamiento, permitirá luego un volver de nuevo al texto revelado con ulteriores y más adecuadas preguntas, y por lo tanto con ulteriores posibilidades de que el mismo libera para nosotros significados e indicaciones ya presentes, pero todavía no suficientemente escuchadas y comprendidas, o a veces incluso ni siquiera individuadas ${ }^{34}$.
\end{abstract}

En este proceso, la significación moral de la revelación no reside en que esta produzca por sí misma un incremento cuantitativo del saber moral en el campo del obrar concreto, sino en sus "implicaciones antropológicas", que inciden en la precomprensión que posee el intérprete de la realidad y de la ley natural, y que por este camino influirán también en los contenidos éticos, imprimiendo a la moral cristiana su fisonomía inconfundible ${ }^{35}$.

Quisiera ilustrar estas afirmaciones a través de dos ejemplos. Uno de ellos es el de la moral sexual. En la Biblia, obviamente, no encontramos un tratamiento explícito y sistemático de la sexualidad, sino más bien una pluralidad de tradiciones, que traslucen visiones distintas y a veces incompatibles entre sí, y textos normativos que reflejan esta variedad y contraste. Por otra parte, hay un conjunto de contenidos (discursos, imágenes, metáforas, etc.) que no se refieren

Sigo en este punto a Sergio Bastianel y Luigi Di Pinto, "Per una fondazione biblica dell'etica", en Tullo Goffi y Giannino Piana (coord.), Corso di morale, vol. I, Vita nuova in Cristo (Brescia: Queriniana, 1990), 75-78. Ibíd., 77.

35 Cf. Klaus Demmer, Interpretare e agire. Fondamenti della morale cristiana (Milano: Paoline, 1989), 77-79. 
explícitamente al tema y pueden ser, sin embargo, de gran importancia. ¿Cómo afrontar toda esta complejidad?

Una posibilidad consiste en comenzar con un análisis fenomenológico, estudiando esta dimensión de nuestra personalidad en sus diferentes niveles (físico, psicológico, personal, social, espiritual). A través de ellos, la sexualidad se presenta básicamente como un impulso de autotrascendencia que es asumido progresivamente en síntesis superiores, y finalizado a la autodonación y la comunión con el otro humano y con el Otro absoluto. A partir de estos resultados, es posible interrogar el texto bíblico, y recibir la interpelación que proviene de él. Contenidos explícitos que revelan una visión negativa, patriarcal o ritualista de la sexualidad mostrarán su total desajuste con la comprensión de su significado auténticamente humano, positivo, interpersonal y confiado a la propia responsabilidad moral. A la inversa, textos no referidos explícitamente a la sexualidad, revestirán un valor fundamental para su comprensión. Las palabras de Jesús en la institución de la Eucaristía, "esto es mi cuerpo", ¿no están reflejando la meta última de la sexualidad humana, vale decir, la entrega del propio cuerpo, de la totalidad de la persona, por amor a los demás y a Dios mismo? La comprensión de la sexualidad a la luz de la autotrascendencia personal queda así confirmada y, a la vez, llevada a su última y radical profundidad ${ }^{36}$.

Otro ejemplo ilustrativo, es el que se está desarrollando en el campo católico en los últimos años sobre el tema de la justicia penal. Si investigamos desde una aproximación temática los textos referidos a esta cuestión encontraremos, dentro de una esperable heterogeneidad, gran cantidad de material que parece abogar por un principio absoluto de retribución. Aun Jesús, que en el Sermón de la Montaña llama a superar la ley del Talión, no vacila en incorporar algunas expresiones violentas en sus enseñanzas, parábolas e imágenes, sin 
condena alguna (por ejemplo, el deudor vendido con toda su familia para pagar deudas, el rey despechado que incendia la ciudad de los invitados reticentes, o que manda decapitar a quienes conspiraron contra él, etc.). Pero la evolución tanto de la doctrina sobre la pena como de la praxis de la justicia penal, han mostrado los límites de la retribución, la inhumanidad de las penas violentas, en especial la pena de muerte, e incluso de la prisión, y han puesto en evidencia la fecundidad de penas alternativas y de enfoques rehabilitadores. Interrogada la Sagrada Escritura a partir de estos datos, es posible descartar con relativa facilidad buena parte de los contenidos culturalmente condicionados y poner en evidencia, por ejemplo, que la justicia divina es justicia salvadora, que en Dios se conjuga la retribución con la misericordia, y que la idea de la justicia escatológica es una instancia crítica formidable contra la ideología de la justicia terrenal absoluta. De este modo se hace posible pensar juntas la retribución y la rehabilitación, en el contexto de una "justicia reconciliadora" de inspiración evangélica pero, a la vez, plenamente racional ${ }^{37}$.

El enfoque utilizado en los dos casos, el de una ininterrumpida circularidad hermenéutica entre la Biblia y otras fuentes de conocimiento, es plenamente compatible con ByM, sobre todo si se incluye en la "convergencia" el aporte de las ciencias (y en verdad no hay motivo para restringir este criterio al aspecto más existencial de la "sabiduría"), y al mismo tiempo, permite superar la ya mencionada unilateralidad del criterio de contraposición.

En todo caso, el método propuesto ByM para el uso de la SE en la teología moral comporta consecuencias de vasto alcance. Por ejemplo, permite evitar la falsa expectativa de que el problema de la indisolubilidad del matrimonio y la licitud del divorcio puedan ser resueltos con solo dilucidar el exacto significado del término pornéia en el evangelio según San Mateo, como parecen entender todavía hoy muchos especialistas. Tal esclarecimiento sería ciertamente impor- 
tante, pero no concluyente, puesto que debería ser confrontado con elementos provenientes de otras fuentes, a los efectos de poder llegar a una conclusión normativa. En esta misma línea, quisiera finalizar esta reflexión presentando, en modo tentativo, algunas conclusiones.

\section{Conclusiones}

La idea de la "moral revelada", central en el documento, indica, conforme a lo que hemos dicho, que la moral cristiana es ante todo una moral religiosa, en cuanto que es una respuesta al don de Dios, a su intervención salvífica en la historia de la humanidad y de cada persona en particular. Al ser vinculados al indicativo de la acción divina, los textos bíblicos de carácter imperativo son leídos bajo una nueva luz, en cuanto incorporados en la dinámica de la Alianza con Dios.

La Biblia no puede ser ya leída como un código moral estático, pero su interpretación tampoco queda librada a un "intuicionismo" piadoso. ByM preserva la racionalidad del método interpretativo a través de una serie de criterios que en su mutua y estrecha referencia son aptos para controlar y encauzar la labor exegética. En el tratamiento de los mismos se encuentra implícita la idea de que el discernimiento a la luz de la Sagrada Escritura requiere un recurso a otras fuentes complementarias: la sabiduría humana, la ciencia, la tradición, etc. Paradójicamente, esta visión viene a coincidir con las conclusiones de J. Gustafson, crítico de la "moral revelada" (en su acepción más corriente que no es obviamente la de ByM):

La Escritura sola nunca es la instancia de apelación final de la ética cristiana. Su comprensión de Dios y sus propósitos, de la condición del hombre y sus necesidades, de preceptos, acontecimientos, relaciones humanas, sin embargo, provee una orientación básica hacia juicios particulares. Dentro de esa orientación muchos y complejos procedimientos y apelaciones son ejercitados, y hay lugar para una gran cantidad de argumentación. La justificación más decisiva para este uso amplio (loose) de la Escritura puede ser expresado del siguiente modo: la vocación de la comunidad cristiana es la de discernir qué es lo que Dios está haciendo posible y requiriendo que el hombre sea y haga en circunstancias naturales, históricas y sociales particulares. Sus juicios morales son hechos a la luz de ese deber o exigencia fundamental. Así, la Escritura informa 
profundamente estos juicios (...) pero no determina por sí misma lo que deben ser. Tal determinación es hecha por las personas y comunidades como agentes morales finitos y responsables ante $\operatorname{Dios}^{38}$.

Pero dicho esto, es preciso señalar cierta contradicción de fondo en el documento. Por un lado, hemos visto cómo él mismo se esfuerza por dar una respuesta cautelosa sobre la posibilidad de encontrar en la Biblia normas e indicaciones morales. Por otro lado, ByM es obra exclusiva (o al menos así parece) de los exégetas de la Pontificia Comisión Bíblica, quienes han afrontado la tarea de estudiar la moral bíblica sin recurrir a la colaboración de los moralistas, precisamente aquellos a quienes este documento está dirigido.

Una consecuencia inocultable de este hecho es la distinción (y por momentos, separación) entre normas apodícticas (formales, fundadas teológicamente y absolutas) y casuísticas (materiales, culturalmente condicionadas y adaptables), que recuerda la discutida distinción entre nivel trascendental y categorial, y que bajo diversas formas, atraviesa todo el documento, sin que el problema de cómo se relacionan ambos tipos de normas en el texto bíblico sea afrontado, y quizás ni siquiera percibido.

El aspecto positivo es que, siempre que no se caiga en una contraposición artificial, esta distinción refuerza la actitud crítica frente a toda pretensión de encontrar en la Sagrada Escritura normas de acción de un modo más o menos directo. En el mismo sentido debe valorarse el hecho de que ByM despliegue su reflexión en un nivel más bien formal, y que desarrolle al mismo tiempo una criteriología tan articulada en orden al discernimiento de los preceptos morales concretos.

Esta opción del documento tiene un gran alcance al momento de pensar los fundamentos bíblicos de la doctrina de los absolutos morales, quizás el tema central en el debate ético posconciliar. La metodología propuesta por ByM implica en sí misma una profunda crítica (es difícil saber hasta qué punto deliberada) al modo como el 
magisterio católico suele utilizar la Sagrada Escritura hasta el día de hoy. No se puede menos que coincidir con R. Gula, cuando afirma que "en la tradición católica, muchos documentos de ética sexual (por ejemplo, Humanae vitae y Persona humana) usan la Escritura como «moral revelada» haciendo referencia explícita a normas absolutas e inmutables, que sirven como fuente primaria o corroborativa de sus posiciones morales" ${ }^{139}$.

Esta observación es aplicable con más razón a Veritatis splendor, encíclica cuyo objetivo central es la afirmación de los absolutos morales. Este documento utiliza ampliamente la Sagrada Escritura al servicio de la afirmación de la doctrina del intrinsece malum. A este objetivo se ordena, en su primer capítulo, tanto la lectura del texto del joven rico (Mt 19) como la reflexión sobre el Decálogo. En ByM, en cambio, casi no se encuentran rastros de esa problemática; por el contrario, las normas universales y permanentes son situadas una y otra vez en el nivel del derecho apodíctico, de carácter formal. De este modo, lejos de avalar el uso de los textos bíblicos en Veritatis splendor, más bien contribuye a poner en evidencia su selectividad y unilateralidad en el recurso a los mismos, con el fin de sostener visiones teológicas preconcebidas (por ejemplo, la vida moral como cumplimiento de los mandamientos con auxilio de la gracia), o de resolver problemáticas ajenas a la Sagrada Escritura (por ejemplo, la discusión sobre el proporcionalismo) ${ }^{40}$.

Que estas implicancias hayan estado en la mente y la intención de los miembros de la Pontificia Comisión Bíblica parece improbable. Pero también es posible que estemos ante una inadvertencia providencial, que preserva a ByM de la tentación de leer la Sagrada Escritura con el prisma de problemáticas que le son ajenas. En

39 Richard Gula, Reason informed by faith: foundations of Catholic morality (Mahwah NJ: Paulist Press, 1989), 169.

40 Cf. Gareth Moore, "Some Remarks on the Use of Scripture in Veritatis Splendor", en Joseph Selling y Jan Jans (coord.), The Splendor of Accuracy. An Examination of the Assertions made by "Veritatis Splendor" (Kampen - the Neetherlands: Pharos, 1994), 71-97; William Spohn, "Morality on the Way of Discipleship", en Michael Allsopp y John O'Keefe (coord.), "Veritatis Splendor". American Responses (Kansas City: Sheed \& Ward, 1995), 83-105. 
cualquier caso, se percibe en este documento un toque de frescura y de audacia que, junto con los otros méritos ya señalados, hacen de él un instrumento de gran utilidad para el trabajo de los moralistas católicos.

\section{Bibliografía}

AA.VV. La giustizia penale e la teologia morale, Rivista di Teologia Morale 138 (2003): 173-175.

Abbà, Giuseppe. Quale impostazione per la filosofia morale? Roma: LAS, 1995.

Artus, Olivier. "Bible et morale. Quels critères pour discerner? Le document de 2008 de la Commission biblique pontificale", Revue d'étique et de théologie morale 260 (2009): 51-68.

Bastianel, Sergio y Di Pinto, Luigi. "Per una fondazione biblica dell'etica". En Tullo Goffi y Giannino Piana (coord.). Corso di morale, vol. I: Vita nuova in Cristo, 75-173. Brescia: Queriniana, 1990.

Bastianel, Sergio. "Autonomía y teonomía". En Francesco Compagnoni y otros (coord.). Nuevo diccionario de teología moral, 124-127. Madrid: Paulinas, 1992.

Comisión Teológica Internacional. En búsqueda de una ética universal: nueva mirada sobre la ley natural. Marzo 27, 2009. www.vatican.va/roman_curia/congregations/cfaith/cti_documents/rc_con_cfaith_doc_20090520_legge-naturale_ sp.html (Consultada en abril 5, 2010).

Curran, Charles. "The Role and Function of the Scriptures in Moral Theology". En Charles Curran y Richard McCormick (coord.). Readings in Moral Theology, vol.4: The Use of Scripture in Moral Theology, 178-212. New York: Paulist Press, 1984. 
De Mingo Kaminouchi, Alberto. "Una teologia morale più «nutrita dalla doctrina della Sacra Scrittura". En Vicenzo Viva y Gabriel Witaszek (coord.). Etica teologica nelle correnti della storia, 11-30. Vaticano: Lateran University Press, 2011.

Demmer, Klaus. Interpretare e agire. Fondamenti della morale cristiana. Milano: Paoline, 1989.

. Christi vestigia sequentes. Roma: PUG, 1995.

Farley, Margaret. Just Love. A framework for Christian Sexual Ethics. London: Continuum, 2008.

Fumagalli, Aristide. "Sacra Scrittura e agire morale. Una proposta teoretica". Studia Moralia 47/4 (2009): 73-94.

Gula, Richard. Reason informed by faith: foundations of Catholic morality. Mahwah NJ: Paulist Press, 1989.

Gustafson, James. "The Changing Use of the Bible in Christian Ethics". En Charles Curran y Richard McCormick (coord.). Readings in Moral Theology, vol. 4: The Use of Scripture in Moral Theology. New York: Paulist Press, 1984.

. "The Place of Scripture in Christian Ethics: A Methodological Study". En Charles Curran y Richard McCormick (coord.). Readings in Moral Theology, T. IV: The Use of Scripture in Moral Theology, 151-177. New York: Paulist Press, 1984.

Himbaza, Innocent; Schenker, Adrian y Edart, Jean-Baptiste. Consideraciones sobre la homosexualidad en la Biblia. Madrid: Palabra, 2008.

Mckeever, Martin. "Il binomio Bibbia-morale: un nodo da sciogliere". En Vicenzo Viva y Gabriel Witaszek (coord.). Etica teologica nelle correnti della storia. Vaticano: Lateran University Press, 2011. 
Moore, Gareth. "Some Remarks on the Use of Scripture in Veritatis Splendor". En Joseph Selling y Jan Jans (coord.). The Splendor of Accuracy. An Examination of the Assertions made by "Veritatis Splendor", 71-97. Kampen - the Neetherlands: Pharos, 1994.

O'Keefe, John. "No Place for Failure? Augustinian Reflections on Veritatis Splendor". En Michael Allsopp y John O'Keefe (coord.). "Veritatis Splendor". American Responses, 16-37. Kansas City: Sheed \& Ward, 1995.

Pablo VI. Discurso a los profesores italianos de Sagrada Escritura, XXII Semana Bíblica Nacional. Septiembre 29, 1972. www.vatican. va/holy_father/paul_vi/speeches/1972/september/documents/ hf_p-vi_spe_19720929_xxii-settimana-biblica_it.html (Consultada en abril 5, 2010).

Piana, Giannino. "Orientamenti di etica sessuale". En Tullo Goffi y Giannino Piana (coord.). Corso di morale, T. II: Diakonia. Etica della persona, 282-377. Brescia: Queriniana, 1990.

Pinckaers, Servais. Las fuentes de la moral cristiana. Pamplona: Eunsa, 1988.

. Ce qu'on ne peut jamais faire. La question des actes intrinsèquement mauvais. Histoire et discussion. Fribourg - Paris: Cerf, 1987.

Pontificia Comisión Bíblica. La interpretación de la Biblia en la Iglesia. Ciudad del Vaticano: Libreria Editrice Vaticana, 1993.

Salzmann, Todd y Lawler, Michael. The Sexual Person. Toward a renewed Catholic Anthropology. Washington D.C.: Georgetown University Press, 2008.

Schüller, Bruno. "Zur theologischen Diskussion über die lex naturalis". Theologie und Philosophie 4 (1966): 481-503. . La fondazione dei giudizi morali. Trad. Paul Renner. Milano: San Paolo, 1997. 
Spohn, William. "Morality on the Way of Discipleship: The Use of Scripture in Veritatis Splendor". En Michael Allsopp y John O'Keefe (coord.). "Veritatis Splendor". American Responses, 83-105. Kansas City: Sheed \& Ward, 1995.

Stock, Klemens. "Biblia y Moral. Las raíces bíblicas del actuar cristiano. El último documento de la Pontificia Comisión Bíblica". Lumen 59 (2010): 3-16.

Viva, Vincenzo. "La scrittura nella manualistica teologico-morale. Tappe storiche e nodi problematici". Studia Moralia 47/4 (2009): 11-38.

Wodka, Adrzej S. "Bibbia e morale: rilievi di un biblista". En Vicenzo

Viva y Gabriel Witaszek (coord.), Etica teologica nelle correnti della storia, 31-38. Vaticano: Lateran University Press, 2011.

Recibido: diciembre de 2011 Arbitrado: febrero de 2012 